PSICOLOGÍA

IBEROAMERICANA
Psicología Iberoamericana ISSN: 1405-0943

revista.psicologia@ibero.mx

Universidad Iberoamericana, Ciudad de México México

\title{
Florecimiento y salud mental óptima en tiempos de COVID-19
}

Barragán Estrada, Ahmad Ramsés

Florecimiento y salud mental óptima en tiempos de COVID-19

Psicología Iberoamericana, vol. 29, núm. 1, 2021

Universidad Iberoamericana, Ciudad de México, México

Disponible en: https://www.redalyc.org/articulo.oa? id=133967526004

DOI: https://doi.org/10.48102/pi.v29i1.244

\section{(c) (1)}

Esta obra está bajo una Licencia Creative Commons Atribución 4.0 Internacional. 
Artículos

\title{
Florecimiento y salud mental óptima en tiempos de COVID-19
}

\author{
Flourishing and Optimal Mental Health in Times of \\ COVID-19 \\ Ahmad Ramsés Barragán Estrada \\ ramsesbarragane@hotmail.com \\ Consultoría ZP, México \\ https://orcid.org/0000-0001-5063-603X
}

Psicología Iberoamericana, vol. 29, núm. 1,2021

Universidad Iberoamericana, Ciudad de México, México

Recepción: 08 Septiembre 2020 Aprobación: 25 Enero 2021

DOI: https://doi.org/10.48102/ pi.v29i1.244

Redalyc: https://www.redalyc.org/ articulo.oa?id=133967526004
Resumen: La pandemia conocida como COVID-19 y surgida en una provincia de China, Wuhan, nos tomó por sorpresa a todos por igual y se volvió el desafío de salud pública más importante en todo el mundo, de formas que nos ocupan a todos los profesionales de la salud y obligándonos a desarrollar competencias profesionales específicas, así como intervenciones clínicas y estrategias de afrontamiento apremiantes. Así, el presente artículo tiene por objetivo ofrecer un panorama general acerca de las condiciones actuales de COVID-19, explicar la importancia de la salud mental en tiempos de la pandemia, proponer al campo de la Psicología Positiva como un modelo válido de intervención y presentar una propuesta de tratamiento por medio de esta aproximación clínica.

Palabras clave: florecimiento, salud mental óptima, COVID-19, Psicología Positiva, intervenciones clínicas primarias.

Abstract: The pandemic known as COVID-19 emerged in a province of China, Wuhan, and took us by surprise. It became the most important public health challenge in the world, in ways that concern all professionals in health and forced us to develop specific professional skills, as well as important clinical interventions and coping strategies. This paper aims to offer a general overview of the current conditions of COVID-19, explaining the importance of mental health during the pandemic. The paper proposes the field of Positive Psychology as a valid model of intervention and a treatment plan following this approach.

Keywords: flourishing, optimal mental health, COVID-19, positive psychology, primary clinical interventions.

\section{Introducción}

Atender problemas que afectan o deterioran la salud mental de las personas, ha sido una de las tareas asiduas de la psicología desde que ésta es ciencia. Asimismo, la psicología clínica ha puesto un notable empeño en la descripción y comprensión del malestar en general y que hemos enmarcado en subsistemas como la psicopatología, o la intervención y rehabilitación de las personas con trastornos mentales y físicos derivados de eventos cotidianos, normales y extraordinarios (Escobedo, 2008). Sin embargo en las dos últimas décadas, también hemos avanzado notablemente en el desarrollo de otros subsistemas interesados en los aspectos saludables de la personas (Mariñelarena-Dondena \& Gancedo, 2011) y en los procesos psicológicos asociados al crecimiento luego de 
acontecimientos traumáticos o experiencias desafortunadas que aquejan a todos por igual. La presente pandemia conocida como COVID-19 entra aquí y de formas que nos ocupan a todos los profesionales de la salud, obligándonos a desarrollar competencias profesionales específicas que posibiliten estrategias de afrontamiento necesarias y apremiantes en tiempos actuales.

El presente artículo tiene por objetivo ofrecer un panorama general acerca de las condiciones actuales de COVID-19, explicar la importancia de la salud mental en tiempos de la pandemia, proponer al campo de la Psicología Positiva como un modelo válido de intervención y presentar una propuesta de tratamiento por medio de esta aproximación clínica. Así, se confía en que a través de la revisión teórica presente, puedan diseñarse en el futuro próximo estrategias de intervención clínicas susceptibles de ser utilizadas por los profesionales de la salud mental durante y después de COVID-19, y basadas en el modelo de la Psicología Positiva.

\section{La pandemia de Covid-19 y sus efectos psicológicos}

Aunque nadie esperaba que el virus SARS-CoV-2, mejor conocido como COVID-19, se convirtiera tan abruptamente en un fenómeno epidemiológico amenazante, catastrófico y sin precedentes, lo cierto es que así ocurrió y en formas que aún seguiremos tratando de comprender y dilucidar. COVID-19 es oficialmente una pandemia: una enfermedad infecciosa relacionada con el síndrome respiratorio agudo por coronavirus 2, que ha afectado a un estimado de 183 países -hasta el momento en que se escriben estas líneas- (Lorenzo et al., 2020). Comenzó en una provincia de China llamada Wuhan en diciembre de 2019 y en menos de un mes se había extendido a 19 países y ocasionando la muerte de 213 personas y casi 12,000 infectados (Adhikari et al., 2020). Incluye síntomas como fiebre, tos, fatiga, neumonía, dolor de cabeza, diarrea, hemoptisis y disnea (aunque recientemente se ha podido observar, además, que el virus muta con facilidad, ocasionando con ello otra variedad de síntomas). Por si fuera poco, lo que hace tan letal al virus SARS-CoV-2 es su capacidad de contagio y veloz propagación, por lo que probablemente sea ya la infección respiratoria de mayor impacto y daño internacional en las últimas décadas (Urzúa et al., 2020). Sus consecuencias han sido devastadoras en todos los sentidos (económicas, políticas, laborales, culturales, etc.) y han colisionado en todas las áreas de vida de las personas en mayor o menor medida, con especial énfasis en la salud física, mental y social.

Ante esta realidad que a todas luces pareciera tan desesperanzadora, también es verdad que el mundo lleva haciéndole frente a pandemias y epidemias a lo largo de la historia y sobretodo en siglos recientes. Por ejemplo, el cólera en el siglo XIX, el VIH-SIDA en los años ochenta, el virus del Ébola en África, el zika en Brasil o el MERS-COV como uno de los más recientes. En otras palabras y refiriéndonos al tema que nos ocupa, hemos aprendido mucho sobre el efecto psicológico que produce 
lidiar con enfermedades mortales como la que hoy vivimos y que se suma a muchas otras que siguen afectando la vida de miles y miles.

Entre estos efectos psicológicos derivados de COVID-19, están las múltiples manifestaciones clínicas asociadas a la ansiedad, la angustia mental, el estrés negativo, los temores irracionales, el miedo a la muerte, la paranoia, la soledad, la frustración, la depresión y diferentes cuadros psicopatológicos de síndromes o trastornos relacionados (por ejemplo, el síndrome de la cabaña o el Trastorno por Estrés Postraumático) (Inchausti et al., 2020). Además, se ha encontrado evidencia de que el virus asociado con el pensamiento sobre la muerte, se relaciona con otro tipo de problemáticas psicológicas, tales como la hipocondriasis, el insomnio, las crisis de ansiedad generalizada y los ataques de pánico (Legido-Quigley et al., 2020).

Bajo esta misma dirección, recordemos que la estrategia general en un gran número de países, fue el distanciamiento social a través de cuarentenas llevadas a cabo en casa y que podían comprender de uno a varios meses de duración. Lo anterior también detona en afectaciones psicológicas que debemos seguir considerando dentro de la ecuación. Diversas investigaciones, por ejemplo, completaron una revisión bibliográfica extensa para ubicar factores estresores derivados del encierro, el aislamiento y la distancia social. Así, se hallaron estresores tales como: peor salud mental, conductas de evasión y enojo, temores sobre la salud propia o la de otros, sensación de experimentar síntomas relacionados con la enfermedad, aburrimiento, pérdida de la rutina, frustración, ira, estrés por las pérdidas, ambivalencia, desorganización, tristeza, sentimiento de soledad y encierro, pesimismo y pensamientos negativos (Brooks et al., 2020; Piña-Ferrer, 2020).

Por otro lado, los datos anteriores documentan los problemas de salud mental en términos generales y con la limitada evidencia disponible derivado de lo reciente que es la enfermedad, pero las consecuencias psicológicas también pueden ser multivariadas si incluimos la variable del tiempo: (1): ante la enfermedad: según Shigemura et al. (2020), las primeras respuestas emocionales negativas se enmarcan entre el miedo y la incertidumbre extremos, que a su vez conducen a percepciones distorsionadas del riesgo y posibles conductas sociales negativas; (2): durante la enfermedad: se incluyen muchos de los efectos psicológicos ya mencionados, resaltando las reacciones de angustia, miedo extremo a la enfermedad, depresión, ansiedad, indignación y conductas de riesgo como mayor uso de alcohol, tabaco y aislamiento social; (3): posterior a la enfermedad: posible aparición de trastornos específicos, tales como TEPT (trastorno por estrés postraumático), trastornos de ansiedad, depresión y somatizaciones (Urzúa et al., 2020).

Sumado a todo lo anterior, está "la exacerbación del daño" y que debemos considerar y no sobreestimar. En este sentido, la psicología nos ha enseñado que respuestas como el miedo son necesarias y adaptativas para los eventos amenazantes como lo es ahora la presente pandemia, pero también es cierto que el miedo crónico o desproporcionado se vuelve dañino y conducente a desórdenes psiquiátricos (Garcia, 2017; Shin \& 
Liberzon, 2010). Aunado a ello, durante una pandemia el número de personas afectadas en salud mental suele ser mayor que las personas afectadas por la propia enfermedad (Reardon, 2015).

\section{La psicología positiva como modelo de aproximación}

Entendemos a la Psicología Positiva como un nuevo paradigma de la psicología que se centra en las experiencias positivas que incluyen el bienestar, la autonomía, la autodeterminación, las emociones positivas, la salud física y los factores que permiten a los individuos y sociedades prosperar y florecer. Aunado a ello, se presenta desde su aparición como un campo de estudio que permite integrar lo que sabemos del malestar con el bienestar, facilitando una mejor comprensión de los fenómenos psicológicos y sin que COVID-19 sea la excepción a la regla. Asimismo, debemos recordar que se concibe a esta ciencia como un complemento de la psicología actual y no como un sustituto (Barragán-Estrada, 2012, 2019). De ahí su importancia y valor como un modelo de aproximación vigente y útil en la crisis presente. Por último y bajo este lente es válido preguntarse: ¿cómo usar esta propuesta para curar las enfermedades mentales derivadas de la pandemia y de forma que se promuevan los aspectos saludables de las personas, que es el eje rector de este campo de estudio?

Ante este escenario es que la Psicología Positiva se está poniendo a prueba como quizás no lo había hecho desde su aparición hace justamente veinte años. El reto para el psicólogo clínico positivo, tal y como refiere Ruini (2017), es equilibrar el énfasis entre los recursos psicológicos con los que cuenta el individuo o paciente, con el conocimiento de los aspectos negativos desde lo que ya sabemos en psicopatología y ramas afines; además, este balance puede variar según la actitud, solicitud, condición objetiva y apoyo social por parte del paciente, por lo que también será algo a considerar y en miras de construir una alianza terapéutica adecuada.

Derivado de lo anterior, es que el cambio social (entendido como este cambio de lente para atender y promover la salud mental desde el enfoque salutogénico de la Psicología Positiva) es sí, un gran reto presente de la Psicoterapia Positiva (PPT por sus siglas en inglés), pero también una gran oportunidad conforme se superen los diferentes obstáculos; pues tal cambio de perspectiva puede movilizar los recursos psicológicos en varios niveles en los que no sólo los pacientes se habrán de beneficiar, sino también las familias y la comunidad. De hecho, estudios recientes respaldan esta tesis, argumentando que: (1) apoyar a las personas a reconocer sus fortalezas personales (concepto de gran relevancia en esta disciplina), desarrollar un estilo de atribución más optimista, perseguir metas significativas y fomentar el crecimiento y la autoexpresión, contribuyen a que las personas participen más activamente en la sociedad; (2), hacer que las personas florezcan (prosperen) a través de intervenciones clínicas positivas es un instrumento valioso para aumentar el bienestar de las comunidades (Ruini, 2017); y (3), que las personas "florecientes" suelen ser más amigables, más optimistas, más efectivas en 
el trabajo y se preocupan más por el bienestar de los demás, lo que en consecuencia los vuelve más sensibles a los problemas sociales (Keyes, 2007).

Wood y Tarrier (2010) se refirieron a este enfoque clínico integrador como "Psicología Clínica Positiva" y sin que esto implicara la necesidad de desarrollar un nuevo fragmento en la psicología clínica tradicional, sino más bien un cambio o reorientación del enfoque, de modo que se prestara igual importancia al funcionamiento óptimo y deficitario de las personas a la hora de tratar cualquier padecimiento o problemática.

Para cerrar este apartado y apelando a estos últimos hallazgos científicos, no sólo parece posible, sino plausible, atender los efectos psicológicos presentes en esta pandemia desde lo que ofrece la Psicología Positiva en cuanto a sus dominios clínicos, tal y como empieza a esbozarse en la investigación científica (García-Álvarez \& Cobo-Rendón, 2020; Rashid \& McGrath, 2020). O dicho de otro modo: sostener el bienestar por medio de todos los aspectos positivos del funcionamiento óptimo de las personas y que se incluyen en la definición y componentes de la salud.

\section{Florecimiento: ¿Qué implica florecer?}

El estudio del florecimiento humano refleja un cambio de paradigma en la investigación concerniente a la salud mental. Tal y como Michalec et al. (2009) expresan, los estados de salud mental óptima no sólo son aquellos en donde hay una ausencia de síntomas negativos conducentes a una psicopatología, sino que incluyen elementos de vida positivos tales como la sensación de llevar una vida significativa y con propósito, así como contar con lazos o vínculos sociales de calidad con otros.

Para florecer, una persona debe presentar todas las características básicas que se enlistan enseguida y al menos 3 de las secundarias: a) características básicas: emociones positivas, compromiso, interés, sentido, propósito; y b) características secundarias: autoestima sana, optimismo, resiliencia, vitalidad, autodeterminación, relaciones positivas.

Nótese además, el énfasis que se hace sobre la afectividad positiva. Bajo esta línea, y considerando el efecto benéfico de las emociones positivas, diversas investigaciones han postulado que la positividad entendida como el cultivo de emociones positivas- es un ingrediente necesario para florecer (Fredrickson, 2001; Fredrickson \& Cohn, 2008). Según Fredrickson y Losada (2005), florecer significa vivir dentro de un rango óptimo del funcionamiento humano, uno que connota bondad, productividad, crecimiento y resiliencia.

De hecho, florecer es una de las grandes metas de la Psicología Positiva y bajo la premisa de que todos contamos con la capacidad como seres humanos de cultivar el bienestar en nuestros propios términos y de acuerdo con nuestros valores, creencias, condiciones y recursos con los que contamos o podemos desarrollar. En investigaciones de este tipo y para ser diagnosticado como "floreciente" en la vida, los individuos deben de exhibir altos niveles en una de las diferentes escalas del bienestar emocional, así como en varias de las escalas del funcionamiento positivo y 
que pueden comprender dominios de vida laborales, escolares, de relación de pareja u otros.

Otros investigadores han propuesto que la persona que florece deberá presentar síntomas "opuestos" a los de las enfermedades mentales. Por ejemplo, toman como ingredientes del florecimiento una lista de diez características positivas y que incluso son la base de una propuesta diagnóstica bajo el respaldo de lo que ofrece este campo de estudio desde su aparición. En la lista se incluyen las características de: competencia, estabilidad emocional, compromiso, significado, optimismo, emoción positiva, relaciones positivas, resiliencia, autoestima sana y vitalidad (Huppert \& So, 2013). De acuerdo con esta categorización, florecer significa presentar al menos ocho de las características positivas y la presencia de mayor afectividad positiva.

Lo anterior quiere decir que los individuos florecientes no sólo están libres de enfermedades mentales, sino que también están llenos de vitalidad emocional y funcionan positivamente en las esferas privada y social de sus vidas. Este modelo continuo de la salud mental óptima, describe la condición de florecimiento como la presencia de altos niveles de bienestar hedónico (asociado al placer), bienestar eudaimónico (psicológico) y bienestar social (Keyes \& Haidt, 2003). Además, este modelo abarca y categoriza desde lo que es una enfermedad mental completa (bajos niveles de bienestar y criterios propios del trastorno mental), la salud mental moderada, el florecer y hasta el estado de languidecimiento, mismo que se caracteriza por un nivel deteriorado de estos aspectos del bienestar, aunque sin sufrir del trastorno mental en cuestión (Lamers et al., 2011).

Bajo este tenor, los beneficios del florecimiento también resultan relevantes para la promoción de la salud mental: estudios recientes han revelado que los adultos con salud mental óptima; es decir, personas sin un desorden mental en los últimos 12 meses y en florecimiento, reportan la menor cantidad de días de trabajo perdidos, un funcionamiento psicosocial más saludable (lo que significa baja sensación de minusvalía, objetivos claros de vida, alta resistencia psicológica y alta capacidad de intimidad o cercanía), menor riesgo de enfermedad cardiovascular, menor número de enfermedades físicas crónicas y menor utilización de la atención médica, y entre otros beneficios (López, 2011).

Finalmente, sea ante COVID-19, desastres naturales, conflictos bélicos o escasez de recursos que conducen a la pobreza (por mencionar algunos), un gran número de personas no habrán de cubrir los criterios de un síndrome o trastorno mental severo. Por ejemplo, de las investigaciones llevadas a cabo por Corey Keyes y de la muestra estudiada en este tipo de escenarios, la mayoría de los adultos (85.9\%) no han tenido un episodio depresivo y de este total, más de la mitad profesa una salud mental moderada (es decir, el 58.7\%); además, igualmente cierto es que tan sólo el 21.6\% cumplen con los criterios arriba expuestos que considera la ciencia para florecer y otros tantos (cerca del 20\%) cumplirán los criterios para el "decaimiento", el cual corresponde con una discapacidad psicosocial sustancial asociado con una pobre salud emocional y altas limitaciones 
de la vida diaria (Keyes, 2010). O dicho de otro modo, la aparición de un trastorno psicológico en tiempos de COVID-19 es probable, la salud mental deteriorada o disminuida (decaimiento o languidecimiento) también; pero la capacidad de florecer y prosperar por medio de los recursos con los que contamos, también y casi con la misma probabilidad.

\section{Salud mental óptima en tiempos del Covid-19}

Por otro lado, ahora sabemos que las personas también traen a terapia sus cualidades, recursos y fortalezas que pueden ser utilizados para resolver sus problemas dentro y fuera del proceso psicoterapéutico. Derivado de lo anterior, una tarea fundamental de cualquier tratamiento es identificar dichos recursos y fomentar su uso, no sólo para el afrontamiento de la adversidad, sino de forma más general con el objetivo de lograr una vida sana y próspera (Park et al., 2013). Este enfoque equilibrado, refieren los mismos autores, conllevará el establecimiento del rapport con el psicólogo y fomentará la confianza de sus clientes. Bajo esta misma línea, distintos enfoques de la terapia familiar sistémica coinciden, por ejemplo al afirmar que construir una sesión de terapia es lograr pasar de las charlas del problema a las charlas de la solución (De Shazer \& Berg, 1992).

Una definición de Psicoterapia Positiva (PPT) que se alinea con la atención a la salud mental en la actualidad y que puede ser uno de los ejes de acción para los problemas psicológicos derivados de COVID-19 es la que proponen Seligman et al. (2006). Seligman et al. (2006) entendida PPT como un tratamiento psicológico que busca reducir los síntomas del funcionamiento negativo mientras contribuye a desarrollar compromiso y el cultivo de emociones positivas. Por ejemplo, diseñando nuevas metas y objetivos de vida, al tiempo que podemos promover la esperanza y el disfrute individual pese al contexto de incertidumbre derivado de la pandemia. Además, parte de los objetivos y premisas fundamentales de este tratamiento, consiste en prestar mayor atención (o por lo menos la misma) a la fortaleza en comparación con la debilidad; equilibrar la balanza con tal de ir del -5 en la vida de las personas al +8 o quizás más arriba; asimismo, "despatologizar" para comenzar a construir los mejores aspectos de la vida de cualquier persona (Barragán-Estrada, 2019). Finalmente, la visión de este modelo orientado a la salud psicológica, implica experimentar mayor satisfacción con la vida, relaciones positivas significativas, contribuciones a la comunidad, cultivar la vida con sentido, y sentirnos sanos y seguros.

\section{Diseñando los primeros planes de intervención}

Atender la salud mental y promover el florecimiento en tiempos de COVID-19 significa diseñar planes de intervención centrados en aumentar el uso de las habilidades y recursos propios de los pacientes para obtener soluciones terapéuticas a sus problemas. Bannink (2014), por ejemplo, sugiere cambiar el foco de la terapia de lo que está mal a lo que 
está bien, y de lo que no funciona a lo que sí. Esta transición representa el cambio de paradigma de la psicología clínica positiva pasando de un enfoque centrado en los déficits y debilidades, a uno que se basa en los recursos y soluciones. Por ejemplo, podemos cuestionar a los pacientes sobre qué fortalezas les han permitido afrontar la pandemia presente o cómo es que cultivan su bienestar en casa o durante el aislamiento social.

Así, también podemos reorientar el trabajo clínico hacia el diseño de metas que para el paciente resulten significativas, del mismo modo que puede aprender a forjar lazos más íntimos y profundos con las personas a través de la distancia social (como puede ser al usar los medios de comunicación digitales para acercarnos a nuestros seres queridos). Esto último es también la esencia de la Psicoterapia Positiva: implementar exitosamente estrategias profundas relacionadas y aplicables al bienestar de los pacientes (Seligman \& Peterson, 2003) y en donde el psicoterapeuta es capaz de identificar y remover los obstáculos que impiden al paciente llevar una vida plena y floreciente.

No obstante, la otra parte de la ecuación es igualmente cierta y debemos tenerla presente. Así, se puede volver muy complicado cultivar positividad (emociones positivas) cuando muchas de estas dependen del contacto físico o de la cercanía con otras personas. En este sentido, primeros aportes de la investigación sugieren que lo anterior es posible y que podemos mantener emociones como el sentido del humor, el júbilo y otras conexiones sociales pese a la distancia física (Yamaguchi et al., 2020). Por ejemplo, diferentes iniciativas han logrado ser implementadas exitosamente, tal y como documentan los mismos autores. Nintendo es una de ellas, al lanzar un juego en línea llamado "Animal Crossing" en donde los jugadores pueden pasear libremente entre las casas de otros para fortalecer la interacción social. De la misma manera, el músico japonés Gen Hoshino (2020) propuso "saborear" aún más su canción "Dancing on the Inside" pidiéndole a la gente que subiese momentos memorables de su vida social editando y manipulando las imágenes y sonidos junto con la canción, lo cual atrajo a un numeroso público en línea que respondió a la iniciativa y mientras se mantenía el confinamiento.

Lo anterior coincide con otro tipo de investigaciones que nos permiten cultivar fortalezas personales de una forma integrativa y pese a la adversidad. Según Peterson y Seligman (2004), nuestros rasgos positivos de personalidad aumentan nuestra capacidad para el disfrute, las gratificaciones y se traducen en estados de felicidad más duraderos. Para ello, las personas pueden utilizar tres estilos de comportamiento relacionados: lo placentero, el sentido de vida y el compromiso. Así, una persona temerosa del contagio o en distanciamiento social puede redirigir su miedo y ansiedad por una serie de actividades en casa donde ponga en marcha estas cualidades pragmáticas y descubrir que el Autocontrol y el Sentido del humor (ejemplos de fortalezas) le son placenteras, le permiten obtener mayores placeres si no desiste de su uso (gratificaciones), y las vuelve parte de su compromiso en razón de que se traducen en logros significativos, permitiendo además que su vida sea una vida con sentido. 
Otra forma de empezar a preparar el camino dentro de los planes de intervención es desarrollando estrategias de afrontamiento contra el estrés, los traumas y las dificultades, tal y como sugiere Lyubomirsky (2011) y de manera que los problemas que atravesamos durante la pandemia no afecten negativamente nuestra existencia. De acuerdo con esta investigadora, las personas podemos trazar estrategias personales que nos permitan sobreponernos mejor a la adversidad. Entre las estrategias más efectivas están: (a) concentrar esfuerzos al respecto de la situación y emprender una acción; no quedarse a esperar la solución; (b) sistematizar las actividades: hacer una sola cosa a la vez; (c) desarrollar un plan de acción; (d) dejar de lado las otras cosas para concentrarse en aquella que ha suscitado la dificultad; (e) escuchar consejos y recomendaciones sobre lo que puedo hacer; ( $\mathrm{f}$ ) hablar con alguien que pueda hacer algo en concreto; (g) replantear las suposiciones y creencias: encontrarle algún sentido a la dificultad o trauma; ( $h$ ) traducir en palabras y enunciados aquello que nos angustia y aflige; por ejemplo, al llevar un diario o escribir acerca de los planes futuros una vez superado el malestar ocasionado por la pandemia.

Para concluir, la literatura experimental ha mostrado que los sujetos que se plantean metas congruentes con sus valores e intereses, se acercan mucho más al bienestar, la autodeterminación y alcanzan con mayor probabilidad las metas planteadas (Vázquez et al., 2006). Incluso y en un contexto más universal, distintas filosofías tanto orientales como occidentales, proponen que el desarrollo de metas que equilibren el desafío con la realidad, promueve en las personas una mejor salud mental y una personalidad mucho más madura (Barragán-Estrada, 2019).

\section{Estrategias e intervenciones clínicas orientadas al florecimiento y la salud mental óptima durante y después de Covid-19}

Tal y como Xiang et al. (2020) sugieren, las estrategias de prevención y promoción de la salud mental ante el escenario de COVID-19, deberán considerar tres factores fundamentales: la conformación de equipos multidisciplinarios que además de psicólogos, incluya otros profesionales de la salud mental tales como psiquiatras, enfermeras y trabajadores sociales; establecer mecanismos de comunicación claros, precisos y actualizados en relación a COVID-19; y el establecimiento de servicios confiables de asesoría psicológica (por ejemplo, a través de dispositivos electrónicos o aplicaciones destinadas a tal fin). Por otro lado -e igualmente importante- es la creación de políticas públicas que atiendan el antes, durante y el después de la pandemia, y que de igual manera, sitúe a los profesionales de la salud mental en los roles principales y al centro del escenario como parte de la estrategia integral (Shultz et al., 2016).

Un tercer aspecto a considerar y que nos permite confiar en que el florecimiento y la salud mental óptima no sólo es una realidad posible, sino deseable, será el hecho de seguir situando en primer plano a la construcción del bienestar psicológico y las variables asociadas a este. 
En este sentido, empiezan a aparecer algunos artículos que documentan cómo el mantener una forma de vida asociada a las emociones positivas como la alegría o la tranquilidad, sumadas a la paciencia y tolerancia, permiten mantener la salud mental ante la crisis de COVID-19 y de acuerdo con los postulados de la Psicología Positiva (Yamaguchi et al., 2020). Aunado a ello, el reconocimiento de nuestros recursos psicológicos en los dominios físico, psicológico y social, junto con el uso y búsqueda de fortalezas personales, permitirán el desarrollo de la resiliencia psicológica (Komazawa \& Ishimura, 2017).

Resultados como los recién descritos son alentadores, sobretodo si consideramos que antes de la crisis pandémica, estas estrategias y metodologías de trabajo ya se venían probando con distintos grupos, enfoques y rigor en los análisis. Por ejemplo, la Terapia del Bienestar, desarrollada por Fava y colaboradores (Berrocal et al., 2008), propone que es posible contrarrestar el malestar psicológico a través de las intervenciones encaminadas a aumentar los niveles de bienestar. Además, sugiere que es posible alcanzar el funcionamiento óptimo y llevar una vida saludable ayudando a la gente a darse cuenta de su verdadero potencial, tal y como se ha documentado a lo largo de este artículo. Es una estrategia psicoterapéutica estructurada, directiva, orientada al problema y basada en un modelo educativo que comprende desde ocho hasta 16 sesiones de trabajo espaciadas semanal o quincenalmente; enfatiza la auto-observación (por medio del registro de conductas en un diario estructurado) y la interacción paciente-terapeuta. El desarrollo de las sesiones se lleva a cabo en tres fases:

(a) Sesiones iniciales: dedicadas a identificar los episodios de bienestar que son parte de la vida del paciente y que son ubicados en un contexto situacional sin importar su frecuencia o duración (el paciente se vale de un diario en donde informa de dichos episodios y los puntúa en una escala de o a 100). En el caso de problemáticas asociadas a COVID-19, los episodios de bienestar pueden ser igualmente registrados y puntuados, recordando y haciendo ver al paciente que de lo que se trata es de mantenernos orientados en el bienestar pese a la situación adversa.

(b) Sesiones intermedias: orientadas a identificar pensamientos y creencias que dan lugar a la interrupción de los episodios de bienestar de forma que se puedan cuestionar, además de que posibilita la identificación de áreas del bienestar psicológico que no están siendo afectadas (p.ej. laboral, social) y en miras de ser reforzadas por medio de actividades (tareas diarias). Además, podemos promover la realización de actividades encaminadas al bienestar, tales como el hacer ejercicio, pasar tiempo de calidad con los seres queridos - presencialmente o a distancia- o incluso actividades encaminadas a la conducta altruista.

(c) Sesiones finales: dirigidas a presentar progresivamente las seis dimensiones del bienestar con base en el modelo propuesto de Carol Ryff (Ryff \& Singer, 2008) que permiten detectar problemas específicos existentes y en aras de promover las interpretaciones alternativas al problema (autoaceptación, crecimiento personal, propósito en la vida, dominio del entorno, autonomía y relaciones positivas). Por ejemplo, 
el dominio del entorno se basa en la idea de que podemos controlar ciertos aspectos situacionales o de las propias circunstancias de vida. Así, podemos enfatizar con el paciente aquellas situaciones que durante y después de COVID-19 sí dependen de él, al igual que la aceptación de las que no (qué está en nuestras manos y qué no).

Finalmente, cabe mencionar que la Terapia del Bienestar se ha probado recientemente como una estrategia de atención efectiva ante la depresión y como un medio en la consecución del bienestar a través del propósito y significado de vida (Fava et al., 2017).

Otro modelo que ha probado su eficacia en fechas recientes y ante diferentes malestares psicológicos, es el Modelo PERMA de Seligman, y que ha buscado integrar el bienestar a través de cinco componentes: positividad (emociones positivas), compromiso y fluidez (engagement, uso y cultivo de las fortalezas personales), relaciones interpersonales positivas, significado (vida con sentido) y consecución de logros (Seligman, 2011). De acuerdo con el autor y padre de la Psicología Positiva, podemos aumentar nuestros niveles de bienestar al tiempo que florecemos como individuos, en la medida que pongamos en práctica cada uno de sus elementos de forma equilibrada. Haciendo alusión a los síntomas e impacto psicológico derivado de COVID-19, se proponen las siguientes estrategias:

\section{Tabla 1}

Tabla 1

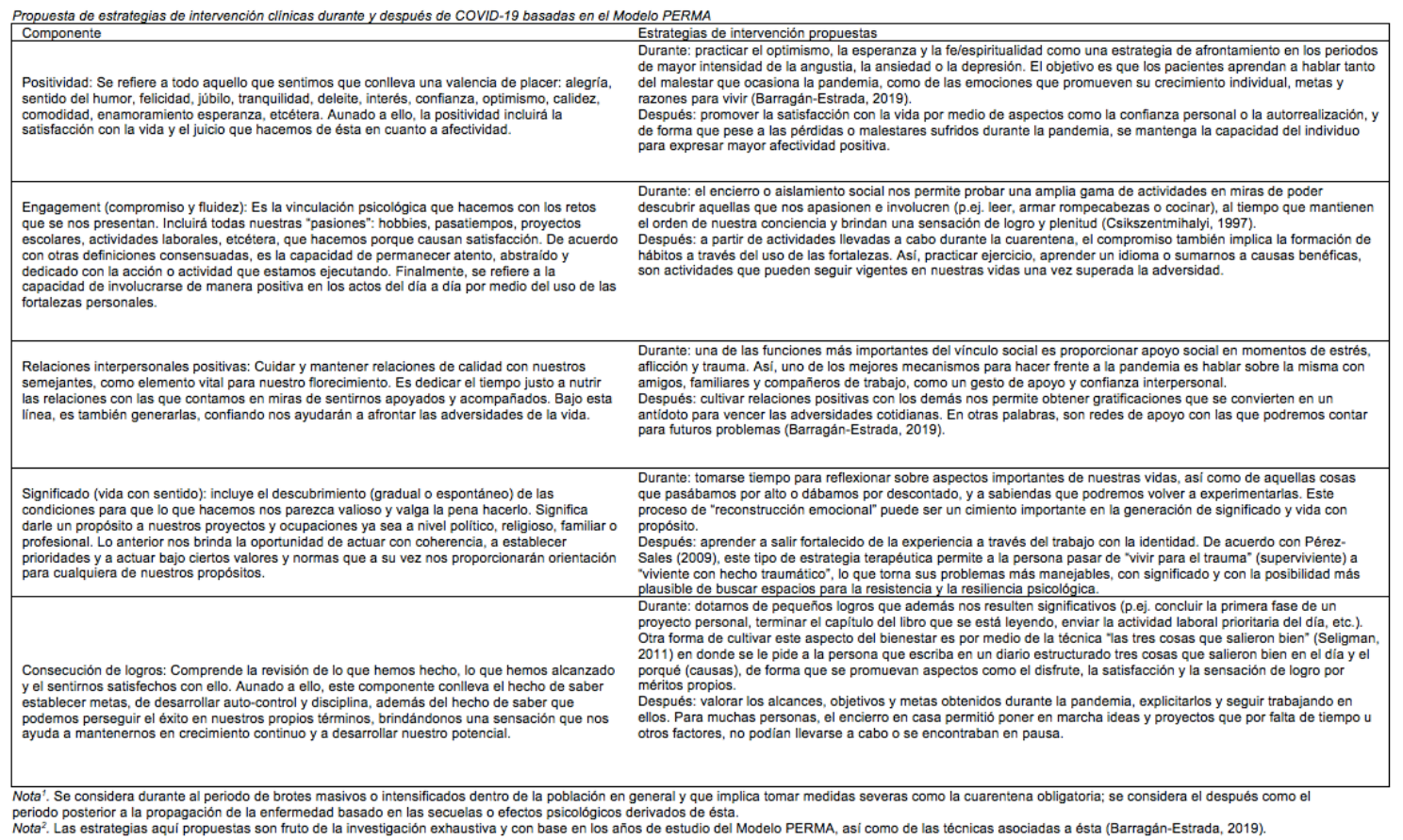

Propuesta de estrategias de intervención clínicas durante y después de COVID-19 basadas en el Modelo PERMA Nota 1. Se considera durante al periodo de brotes masivos o intensificados dentro de la población en general y que implica tomar medidas severas como la cuarentena obligatoria; se considera el después como el periodo posterior a la propagación de la enfermedad basado en las secuelas o efectos psicológicos derivados de ésta.

Nota 2. Las estrategias aquí propuestas son fruto de la investigación exhaustiva y con base en los años de estudio del Modelo PERMA, así como de las técnicas asociadas a ésta (Barragán-Estrada, 2019). 
Un tercer grupo de estrategias e intervenciones clínicas enmarcadas en la capacidad de florecer en épocas de crisis, las encontramos en el modelo de psicoterapia positiva en situaciones adversas (trauma, pérdida o crisis), propuesto por Pérez-Sales (2009). Este modelo, aunado a los anteriores, pone el foco en aspectos del bienestar, tales como cultivo de las emociones positivas, desarrollo, crecimiento personal y significado de vida (entre otros); que de acuerdo con el autor, deben ponerse en juego desde el inicio de la terapia. Por ejemplo, la postura indagatoria inicial conlleva elementos de análisis como la actitud de búsqueda de significado y la distancia de seguridad protectora al situarse el paciente como observador externo de uno mismo. En este sentido y en relación a COVID-19, podemos solicitar al paciente que haga una reflexión profunda de sí mismo durante la crisis vigente, al tiempo que aprende a observarse "desde fuera” en aras de responder gradualmente a las preguntas: ¿quién era yo entonces? y ¿quién soy ahora?

Parte de las estrategias e intervenciones clínicas de este modelo, se centran en la construcción de un mapa identitario a través de la resistencia psicológica y en donde se aprende a contar historias de redención, triunfo o con la capacidad de salir fortalecido (como se mencionó en la tabla anterior, al pasar de ser "sufriente" a viviente con hecho traumático).

Lorenzo et al. (2020) además, postulan que algunas de las acciones futuras necesarias por parte de los profesionales de la salud son: (1) profundizar en los componentes psicológicos y sociales de COVID-19, (2) profundizar en el análisis del proceso salud-enfermedad tanto de quienes padecieron la enfermedad como de los involucrados, (3) promover la investigación relacionada con el distanciamiento social y el aislamiento, (4) delimitar aspectos en común de situaciones adversas similares y (5) sistematizar las prácticas de actuación psicológica, así como el desarrollo de protocolos de atención de la salud, desde la psicología (Lorenzo et al., 2020).

\section{Discusión}

Nadie puede negar que el virus SARS-CoV-2 nos tomó por sorpresa y se volvió el desafío de salud pública más importante en todo el mundo en los últimos años. Con más de 94 milllones de casos en el momento que se escribe esto y más de dos millones de muertes (Johns Hopkins University, 2020), la pandemia de COVD-19 está lejos de terminar aún y cuando las prometedoras vacunas estén llegando ya. Tal y como la mayoría hemos vivido, lejos o cerca de la enfermedad, cambió muchos de nuestros comportamientos habituales, ya sea en lo individual o lo social. En algunos casos acercó a las familias y en otros las alejó; en muchas regiones afectó los empleos y los ingresos, y en unos pocos los mejoró y eficientó; en un número aún indeterminado dañó y deterioró la salud mental presente, aunque creemos que también en muchos otros la fortaleció; etcétera. En cualquiera de los casos y con base en la evidencia aquí presentada derivada de crisis, situaciones adversas y enfermedades anteriores, la salud física, 
mental y social se ha puesto en juego y con ello aparecemos nuevamente en la mira todos los profesionales de la salud relacionados con este virus.

En relación a nuestros objetivos, se ofrece un panorama general en este artículo que permite enfatizar la importancia de la salud mental en tiempos de COVID-19. En adición, se logra aterrizar en lo general, un esquema de tratamiento básico para esta enfermedad y desde la postura clínica de la Psicología Positiva, por lo que confiamos que pese a las limitaciones aquí enunciadas, el artículo sea también una posible herramienta de trabajo para los profesionales de la salud mental.

Finalmente y como podemos observar, la labor es vasta, compleja y en diferentes sentidos, aún incierta. No obstante, la Psicología en general ya se ha enfrentado a desafíos de estas magnitudes en el pasado y hemos aprendido mucho al respecto. La clave es no desestimar los riesgos asociados, garantizar una adecuada atención psicológica, trabajar en conjunto con todos los actores involucrados y contar con protocolos de actuación respaldados por la investigación. Tal y como documentan Inchausti et al. (2020), China por ejemplo, careció de una planificación adecuada en torno a las intervenciones psicológicas; mismas que fueron implementadas desorganizada e ineficazmente. Además, debemos recordar que las intervenciones psicológicas deben darse hasta que la persona lo solicita o existe una marcada sintomatología presente, tal y como la evidencia lo muestra (National Institute for Health and Care Excellence [NICE], 2018). Así, el florecimiento y la salud mental óptima pueden y deben darse de formas naturales y con base en los propios recursos con que cuenta la persona para su afrontamiento. En otras palabras, creemos que somos capaces de florecer aún en la adversidad, ya sea por medios propios o bajo la guía y apoyo de la Psicología. COVID-19 no será la excepción.

\section{Referencias}

Adhikari, S. P., Meng, S., Wu, Y., Mao, Y., Ye, R., Wang, Q., ... \& Zhou, H. (2020). Epidemiology, causes, clinical manifestation and diagnosis, prevention and control of coronavirus disease (COVID-19) during the early outbreak period: A scoping review. Infectious Diseases of Poverty, 9(29). https://doi.org/10.1186/s40249-020-00646-x

Bannink, F. (2014). Post traumatic success: Positive psychology \& solution-focused strategies to help clients survive \& thrive. WW Norton \& Company.

Barragán-Estrada, A. R. (2012). Psicología positiva y humanismo: premisas básicas y coincidencias en los conceptos. Revista Electrónica de Psicología Iztacala, 15(4), 1512-1531.

Barragán-Estrada, A. R. (2019). Psicología del bienestar: guia de intervenciones clínicas, educativas y organizacionales. Editorial Académica Española.

Berrocal, C., Ruini, C., \& Fava, G. A. (2008). Aplicaciones clínicas de la terapia del bienestar. En G. Hervás \& C. Vazquez (Eds.), Psicología positiva aplicada (pp. 101-127). Desclée de Brouwer.

Brooks, S. K., Webster, R. K., Smith, L. E., Woodland, L., Wessely, S., Greenberg, N., \& Rubin, G. J. (2020). The psychological impact of 
quarantine and how to reduce it: Rapid review of the evidence. The Lancet, 395(10227), 912-920. https://doi.org/10.1016/S0140-6736(20)304608

Csikszentmihalyi, M. (1997). Finding flow: The psychology of engagement with everyday life. Basic Books.

De Shazer, S., \& Berg, I. K. (1992). Doing therapy: A post - structural re vision. Journal of Marital and Family Therapy, 18(1), 71-81. https://doi. org/10.1111/j.1752-0606.1992.tb00916.x

Escobedo, P. S. (2008). Psicología clínica. El Manual Moderno.

Fava, G. A., Cosci, F., Guidi, J., \& Tomba, E. (2017). Well-being therapy in depression: New insights into the role of psychological well-being in the clinical process. Depression and Anxiety, 34(9), 801-808. https://doi.org/ $10.1002 /$ da.22629

Fredrickson, B. L. (2001). The role of positive emotions in positive psychology: The broaden-and-build theory of positive emotions. American Psychologist, 56(3), 218-226. https://psycnet.apa.org/doi/10.1037/0003066X.56.3.218

Fredrickson, B. L., \& Cohn, M. A. (2008). Positive emotions. En M. Lewis, J. M. Haviland-Jones \& L. F. Barrett (Eds.), Handbook of emotions (pp. 777796). The Guilford Press.

Fredrickson, B. L., \& Losada, M. F. (2005). Positive affect and the complex dynamics of human flourishing. American Psychologist, 60(7), 678-686. h ttps://doi.org/10.1037/0003-066X.60.7.678

García-Álvarez, D., \& Cobo-Rendón, R. (2020). Aportes de la psicología positiva a la salud mental frente a la pandemia por COVID-19. CienciAmérica, 9(2), 268-276. http://dx.doi.org/10.33210/ca.v9i2.323

Garcia, R. (2017). Neurobiology of fear and specific phobias. Learning \& Memory, 24(9), 462-471. https://doi.org/10.1101/lm.044115.116

Hoshino, G. (2020). I was staying at home and made a song like this. "Dancing on the Inside" [Título traducido] [Video]. Instagram. https://www.instag ram.com/p/B-fFPKrBc-X/ ?hlja

Huppert, F. A., \& So, T. T. (2013). Flourishing across Europe: Application of a new conceptual framework for defining well-being. Social Indicators Research, 110(3), 837-861. https://doi.org/10.1007/s11205-011-9966-7

Inchausti, F., García Poveda, N. V., Prado Abril, J., \& Sánchez Reales, S. (2020). La psicología clínica ante la pandemia COVID-19 en España. Clínica y Salud, 31(2), 105-107. http://dx.doi.org/10.5093/clysa2020a11

Johns Hopkins University. (2020). Dashboard by the Center for Systems Science and Engineering (CSSE) at Johns Hopkins University. https://coronaviru s.jhu.edu/map.html

Keyes, C. L. M. (2007). Promoting and protecting mental health as flourishing: A complementary strategy for improving national mental health. American Psychologist, 62(2), 95-108. https://doi.org/10.1037/0 003-066X.62.2.95

Keyes, C. L. M. (2010). Flourishing. En Corsini encyclopedia of psychology. John Wiley \& Sons. https://doi.org/10.1002/9780470479216.corpsy0363

Keyes, C. L. M., \& Haidt, J. (2003). Flourishing: Positive psychology and the life well-lived. American Psychology Association. 
Komazawa, A., \& Ishimura, I. (2017, Septiembre 30). The relationship between strengths awareness and resilience. 19th Annual Meeting of the Japanese Psychological Academy for Human Care, Yamanashi, Japan.

Lamers, S. M., Westerhof, G. J., Bohlmeijer, E. T., ten Klooster, P. M., \& Keyes, C. L. (2011). Evaluating the psychometric properties of the mental health continuum - short form (MHC - SF). Journal of Clinical Psychology, 67(1), 99-110. https://doi.org/10.1002/jclp.20741

Legido-Quigley, H., Mateos-García, J. T., Campos, V. R., Gea-Sánchez, M., Muntaner, C., \& McKee, M. (2020). The resilience of the Spanish health system against the COVID-19 pandemic. The Lancet Public Health, 5(5), e251-e252. https://doi.org/10.1016/S2468-2667(20)30060-8

López, S. J. (Ed.). (2011). The encyclopedia of positive psychology. John Wiley \& Sons.

Lorenzo, R. A., Díaz, K., \& Zaldivar, P. D. (2020). La psicología como ciencia en el afrontamiento a la COVID-19: apuntes generales. Anales de la Academia de Ciencias de Cuba, 10(2).

Lyubomirsky, S. (2011). La ciencia de la felicidad. Ediciones Urano.

Mariñelarena-Dondena, L., \& Gancedo, M. (2011). La psicología positiva: su primera década de desarrollo. Diálogos. Revista Científica de Psicología, Ciencias Sociales, Humanidades y Ciencias de la Salud, 2(1), 67-77.

Michalec, B., Keyes, C. L. M, \& Nalkur, S. (2009). Flourishing. En S. J. Lopez (Ed.), The encyclopedia of positive psychology (pp. 391-394). Wiley Blackwell.

National Institute for Health and Care Excellence (NICE). (2018). Posttraumatic stress disorder. NICE guideline. www.nice.org.uk/guidance/ ng116 (Error 1: El enlace externo www.nice.org.uk/guidance/ ng116 debe ser una URL) (Error 2: La URL www.nice.org.uk/guidance/ ng116 no esta bien escrita)

Park, N., Peterson, C., \& Sun, J. K. (2013). La psicología positiva: investigación y aplicaciones. Terapia Psicológica, 31(1), 11-19.

Pérez-Sales, P. (2009). Psicoterapia positiva en situaciones adversas. En C. Vazquez \& G. Hervás, G. (Eds.), Psicologia positiva aplicada (pp. 155-190). Biblioteca de Psicología Desclée de Brouwer.

Peterson, C., \& Seligman, M. E. P. (2004). Character strengths and virtues: $A$ handbook and classification. American Psychological Association.

Piña-Ferrer, L. (2020). El COVID 19: Impacto psicológico en los seres humanos. Revista Arbitrada Interdisciplinaria de Ciencias de la Salud: Salud y Vida, 7(4), 188-199. https://doi.org/10.35381/s.v.v4i7.670.

Rashid, T., \& McGrath, R. (2020). Strengths-based actions to enhance wellbeing in the time of COVID-19. International Journal of Wellbeing, 10(4), 113-132. https://doi.org/10.5502/ijw.v10i4.1441

Reardon, S. (2015). Ebola's mental-health wounds linger in Africa: Healthcare workers struggle to help people who have been traumatized by the epidemic. Nature, 519(7541), 13-15. https://doi.org/10.1038/519013a

Ruini, C. (2017). Positive psychology in the clinical domains: Research and practice. Springer.

Ryff, C. D., \& Singer, B. H. (2008). Know thyself and become what you are: A eudaimonic approach to psychological well-being. Journal of Happiness Studies, 9(1), 13-39. https://doi.org/10.1007/s10902-006-9019-0 
Seligman, M. E. P. (2011). Florecer: La nueva psicología positiva y la búsqueda del bienestar. Editorial Oceano.

Seligman, M. E. P., \& Peterson, C. (2003). Positive clinical psychology. En L. G. Aspinwall \& U. M. Staudinger (Eds.), A psychology of human strengths: Fundamental questions and future directions for a positive psychology (pp. 305-317). American Psychological Association. https://doi.org/10.1037 /10566-021

Seligman, M. E., Rashid, T., \& Parks, A. C. (2006). Positive psychotherapy. American Psychologist, 61(8), 774-788.

Shigemura, J., Ursano, R. J., Morganstein, J. C., Kurosawa, M., \& Benedek, D. M. (2020). Public responses to the novel 2019 coronavirus $(2019-\mathrm{nCoV})$ in Japan: Mental health consequences and target populations. Psychiatry and Clinical Neurosciences, 74(4), 281-282. https://doi.org/10.1111/pcn .12988

Shin, L. M., \& Liberzon, I. (2010). The neurocircuitry of fear, stress, and anxiety disorders. Neuropsychopharmacology, 35(1), 169-191. https://doi.org/10. 1038/npp.2009.83

Shultz, J. M., Cooper, J. L., Baingana, F., Oquendo, M. A., Espinel, Z., Althouse, B. M., ... \& Mazurik, L. (2016). The role of fear-related behaviors in the 2013-2016 West Africa Ebola virus disease outbreak. Current Psychiatry Reports, 18(11), 104. https://doi.org/10.1007/s11920-016-0741-y

Urzúa, A., Vera-Villarroel, P., Caqueo-Urízar, A., \& Polanco-Carrasco, R. (2020). La psicología en la prevención y manejo del COVID-19. Aportes desde la evidencia inicial. Terapia Psicológica, 38(1), 103-118. http://dx.d oi.org/10.4067/S0718-48082020000100103

Vázquez, C., Hervás, G., \& Ho, S. (2006). Intervenciones clínicas basadas en la psicología positiva: fundamentos y aplicaciones. Psicología Conductual, 14(3), 401-432.

Wood, A. M., \& Tarrier, N. (2010). Positive clinical psychology: A new vision and strategy for integrated research and practice. Clinical Psychology Review, 30(7), 819-829. https://doi.org/10.1016/j.cpr.2010.06.003

Xiang, Y. T., Yang, Y., Li, W., Zhang, L., Zhang, Q., Cheung, T., \& Ng, C. H. (2020). Timely mental health care for the 2019 novel coronavirus outbreak is urgently needed. The Lancet Psychiatry, 7(3), 228-229. https: //doi.org/10.1016/S2215-0366(20)30046-8

Yamaguchi, K., Takebayashi, Y., Miyamae, M., Komazawa, A., Yokoyama, C., \& Ito, M. (2020). Role of focusing on the positive side during COVID-19 outbreak: Mental health perspective from positive psychology. Psychological Trauma: Theory, Research, Practice, and Policy, 12(S1), S49-S50. http://dx.doi.org/10.1037/tra0000807 